\title{
Cartographie de la biomasse forestière et évaluation du carbone séquestré dans la forêt classée de l'Ouémé supérieur au Centre - Bénin
}

\author{
Mama DJAOUGA $^{1 *}$, Soufiyanou KARIMOU ${ }^{1 *}$, Ousséni AROUNA ${ }^{2}$, \\ Soufouyane ZAKARI ${ }^{1}$, Augustin OROU MATILO ${ }^{3}$, Ismaïla TOKO IMOROU ${ }^{1}$, \\ Ibouraïma $\mathrm{YABI}^{4}$, Julien DJEGO ${ }^{5}$, Omer THOMAS ${ }^{1}$ et Christophe HOUSSOU ${ }^{4}$ \\ ${ }^{1}$ Laboratoire de Cartographie, (LaCarto), Université d'Abomey-Calavi (UAC), 10 BP 1082 \\ Cotonou, Cadjèhoun, Bénin. \\ ${ }^{2}$ Laboratoire de Géosciences, de l'Environnement et Applications (LaGEA), Université Nationale des Sciences, \\ Technologies, Ingénierie et Mathématiques (UNSTIM), 03 BP 304 Abomey, Bénin. \\ ${ }^{3}$ Centre d'Etudes, de Recherches et de Formation Forestières (CERF), Direction Générale des Eaux, \\ Forêts et Chasse (DGEFC), 06 BP 3126 Cotonou, Bénin. \\ ${ }^{4}$ Laboratoire Pierre PAGNEY, "Climat, Eau, Ecosystèmes et Développement" (LACEEDE), \\ UAC, BP 922, Abomey-Calavi, Bénin. \\ ${ }^{5}$ Laboratoire d'Écologie Appliquée (LEA), UAC, 01 BP 526 Cotonou, Bénin. \\ *Auteur correspondant ; E-mail : maloud75@gmail.com / karimoubares@gmail.com; Tél.: (+229) 96204649
}

\section{REMERCIEMENTS}

Les auteurs remercient le Fonds National de la Recherche Scientifique et de l'Innovation Technologique (FNRSIT) pour avoir financé le présent travail à travers le projet cartographie de la biomasse forestière et évaluation du carbone séquestré par les aires protégées du Bénin (Projet PCB).
Received: 05-08-2021
Accepted: 29-11-2021
Published: 31-12-2021

\section{RÉSUMÉ}

La forêt classée de l'Ouémé supérieur au Centre-Bénin est un potentiel puits de carbone en dépit des pressions d'origines anthropiques qui menacent la plupart des écosystèmes forestiers. Cette recherche visait à cartographier la biomasse forestière et le stock de carbone séquestré par les formations végétales de la forêt classée de l'Ouémé supérieur au Bénin. A cet effet, un inventaire forestier a été réalisé dans 73 placeaux circulaires de $18 \mathrm{~m}$ de rayon suivant le protocole de l'Inventaire Forestier National (IFN). Les principales données collectées étaient le diamètre et la hauteur des arbres. Les images landsat OLI-TIRS de 2018 à travers le NDVI ont été utilisées en combinaison avec les données de l'inventaire forestier in situ pour la spatialisation de la biomasse et du carbone séquestré. Les résultats obtenus ont révélé que la biomasse totale produite par les arbres dans la forêt classée de l'Ouémé supérieur, avoisine 13035694 tonnes avec une quantité totale de carbone séquestrée évaluée à 6348383 tonnes. La distribution spatiale de la biomasse et du carbone ont montré de plus fortes quantités de biomasse et de carbone séquestré à l'intérieur de la forêt classée comparativement aux périphéries surtout les zones qui sont situées le long des routes aménagées. Il est alors important d'entreprendre les actions de conservation des formations forestières en vue d'accroître leur potentiel de séquestration de carbone dans l'atténuation des effets des changements climatiques au Bénin.

(C) 2021 International Formulae Group. All rights reserved.

Mots clés : Carbone séquestré, inventaire forestier, NDVI, changement climatique, forêt classée de l'Ouémé supérieur au Bénin. 


\title{
Mapping of forest biomass and assessment of sequestered carbon in the forest reserve of upper Ouémé in Central Benin
}

\begin{abstract}
The Upper Ouémé gazetted forest in Central Benin is a potential carbon sink despite the anthropogenic pressures that threaten most forest ecosystems. This research aims to map the forest biomass and carbon stock sequestered by plant communities in the Upper Ouémé classified forest in Benin. To this end, a forest inventory was carried out in 73 circular plots of $18 \mathrm{~m}$ radius following the protocol of the National Forest Inventory (IFN). The main data collected were tree diameter and height. The 2018 OLI-TIRS landsat images through NDVI were used in combination with the in situ forest inventory data for the spatialization of biomass and sequestered carbon. The results obtained show that the total biomass produced by trees in the Upper Ouémé classified forest is around 13035694 tonnes with a total amount of sequestered carbon estimated at 6348383 tonnes. The spatial distribution of biomass and carbon shows higher amounts of biomass and carbon sequestered inside the gazetted forest compared to the periphery, especially in areas that are located along developed roads. It is therefore important to undertake conservation actions in forest in order to increase their carbon sequestration potential in mitigating the effects of climate change in Benin.
\end{abstract}

(C) 2021 International Formulae Group. All rights reserved.

Keywords: Sequestered carbon, forest inventory, NDVI, climate change, upper Ouémé classified forest in Benin.

\section{INTRODUCTION}

Les forêts concentrent environ $80 \%$ des stocks globaux de carbone ligneux aérien et jouent un rôle important dans le cycle du carbone global (Houghton, 2005 ; Laporte et al., 2010). L'augmentation de la concentration des gaz à effet de serre (GES) dans l'atmosphère, comme le dioxyde de carbone $\left(\mathrm{CO}_{2}\right)$, le méthane $\left(\mathrm{CH}_{4}\right)$ et le protoxyde d'azote $\left(\mathrm{N}_{2} \mathrm{O}\right)$, participe au réchauffement climatique actuel à la surface de la terre. Cette situation préoccupe bon nombre de chercheurs à l'échelle internationale (Loubota Panzou et al., 2016) et se traduit par la signature de plusieurs accords, conférences et conventions comme la convention cadre des Nations - Unies pour les Changements Climatiques (CCNUCC), la conférence des parties (CoP21) à Paris en 2015 sur le climat (Pascal et Peter, 2015).

En effet, dans les écosystèmes forestiers, le carbone est stocké dans la biomasse vivante (biomasse aérienne et biomasse souterraine), le bois mort, la matière organique du sol et la litière (GIEC, 2003 ; Loubota Panzou et al., 2016). En dehors de leurs rôles de conservation de la biodiversité, d'approvisionnement en services écosystémiques, la capacité de ces écosystèmes forestiers à piéger le carbone de l'atmosphère et à le stocker est essentiel pour la mise en œuvre des stratégies d'atténuation des changements climatiques et notamment le mécanisme de réduction des émissions dues à la déforestation et à la dégradation (IPCC, 2007 ; FAO, 2009). Dans cette logique, plusieurs travaux de recherche ont porté sur les estimations de la biomasse forestière et le potentiel de séquestration de carbone des écosystèmes de par le monde (Feldpausch et al., 2012; Mugasha et al., 2013 ; Fayolle et al., 2013 ; Ngomanda et al., 2014 ; Chave et al., 2014).

La plupart de ces travaux repose sur les méthodes basées sur les parcelles des inventaires forestiers utilisant des équations allométriques pantropicales (Brown et al., 1989 ; Chave et al., 2004 ; Feldpausch et al., 2012 ; Chave et al., 2014) et tropicales (Kuyah et al., 2012a ; Mugasha et al., 2013 ; Fayolle et al., 2013 ; Ngomanda et al., 2014). Grace à ces méthodes, il est possible de combiner les données de la télédétection pour spatialiser les estimations de la biomasse forestière et le stock de carbone afin de produire des cartes à différentes échelles (Goetz et al., 2009 ; Clark et al., 2012 ; Bastin et al., 2014). 
Au Bénin, les travaux réalisés portent sur l'estimation de la biomasse aérienne et du stock de carbone des arbres de Lophira lanceolata (Adjéran et al., 2020), l'estimation $\mathrm{du}$ stock de carbone organique dans les plantations d'Acacia auriculiformis (Kooke et al., 2019), la modélisation des stocks de carbone du sol (Guendehou et al., 2014) ; l'établissement $\mathrm{du}$ bilan carbone des mangroves des zones humides (Ajonina, 2013) ; l'évaluation du stock de carbone dans les systèmes agroforestiers à Vitellaria paradoxa et à Parkia biglobosa en région soudanienne du Bénin (Saïdou et al., 2012). Peu de travaux de recherche se sont focalisés sur les données combinées de terrain in situ et de la télédétection pour la spatialisation de la biomasse forestière et du stock de carbone au Bénin. De telle méthode peut permettre de détecter la variation de la contribution des écosystèmes forestiers à la séquestration du carbone dans l'espace et dans le temps au regard de l'augmentation de la concentration atmosphérique en gaz à effet de serre induite par les activités humaines.

La forêt classée de l'Ouémé supérieur au Centre-Bénin, est un potentiel puits de carbone en dépit des pressions d'origine anthropique qui menacent la plupart des écosystèmes forestiers. Elle est caractérisée par une diversité de formations végétales et représente ainsi un cadre idéal pour les recherches scientifiques. L'objectif de cette recherche était de cartographier la biomasse forestière et le stock de carbone de la forêt classée de l'Ouémé supérieur dans le cadre de la mise en œuvre d'un projet de réduction des émissions de gaz à effet de serre liées à la déforestation et à la dégradation des écosystèmes forestiers (REED+).

\section{MATÉRIEL ET MÉTHODES Milieu d'étude}

La forêt classée de l'Ouémé supérieur est située au nord Bénin dans la zone de transition soudano-guinéenne (White, 1986 ; Adomou et al., 2011). Géographiquement, elle est localisée entre les parallèles $9^{\circ} 11^{\prime}$ et $9^{\circ} 47^{\prime}$ de latitude nord et entre les méridiens $1^{\circ} 58^{\prime}$ et $2^{\circ} 28^{\prime}$ de longitude est et se trouve à cheval entre les communes de Djougou (Département de la Donga), de N'Dali et de Tchaourou (Département du Borgou) (Figure 1). Cette forêt a été classée par l'administration coloniale sur une superficie de 177542 ha selon l'arrêté $\mathrm{n}^{\circ} 4310 \mathrm{SE}$ du 27 juillet 1952. Le climat est de type tropical sec et est caractérisé par une succession dans l'année de deux saisons dont une saison des pluies et une saison sèche de durée approximativement égale avec une pluviosité annuelle d'environ $1200 \mathrm{~mm}$. Les types de formations végétales qu'on y rencontre sont diversifiés mais on note une absence de forêts denses humides semidécidues et un enrichissement des forêts claires et des formations savanicoles soudaniennes. La faune abritant cette forêt est variée et diversifiée.

\section{Méthodes de collecte des données Dispositif expérimental}

Un échantillonnage a été réalisé dans les placeaux permanents de $18 \mathrm{~m}$ de rayon précédemment installés pour le compte de l'Inventaire Forestier National en 2006 (IFN, 2006). L'inventaire systématique par échantillonnage statistique terrestre sur la base d'une grille de $15 \mathrm{~km}$ x $15 \mathrm{~km}$, basée sur la projection «UTM » de l'ellipsoïde de référence WGS 1984, Zone 31N, a été retenu. A chaque sommet de cette grille, se trouve une unité d'échantillonnage permanente (Figure 2). Les unités d'échantillonnage non permanent ont été choisies dans un rayon de $1 \mathrm{~km}$ du placeau permanent. Ces placeaux permanents et non permanents de l'IFN (2006) ont été complétés par des placeaux de mêmes dimensions installés de façon aléatoires. Au total 73 placeaux ont été installés (Tableau 1).

\section{Données collectées}

Deux catégories de données ont été collectées. Il s'agit des données planimétriques et celles d'inventaire forestier. Les données planimétriques concernent les images landsat 
OLI-TIRS de 2018. Elles ont été obtenues à travers le lien www.earthexplorer.usgs.gov. En ce qui concerne les données d'inventaire, elles ont été collectées dans les placeaux précédemment installés et ont concerné le type de formation végétale en place, le diamètre $(\mathrm{DBH} \geq 10 \mathrm{~cm})$ et la hauteur des arbres respectivement avec le ruban pi et le clisimètre. Dans le cas où il y a des contreforts ou des racines échasses, le diamètre a été mesuré audessus d'eux en fonction des recommandations faites par Clark et al. (2001).

\section{Traitement des données}

Méthodes d'estimation de la biomasse forestière

La biomasse totale $\left(\mathrm{B}_{\mathrm{T}}\right)$ est la somme de la biomasse aérienne $\left(\mathrm{B}_{\mathrm{a}}\right)$ et de la biomasse souterraine $\left(B_{r}\right)$ de l'ensemble des individus inventoriés. Elle a été d'abord estimée par placeau puis par unité de végétation après identification des placeaux spécifiques à chacune d'elle. La biomasse totale a été déterminée en sommant les quantités totales de biomasse obtenue pour chaque unité de végétation. Par souci de simplification, les résultats ont été présentés en tonnes par hectare.

$$
B_{T}=\left\{\begin{array}{c}
B_{a}+B_{r} \\
\text { ou } \\
B_{a}(1+R)
\end{array} \text { Avec } B_{r}=B_{a} * R\right.
$$

$\mathrm{R}$ : facteur de conversion de la biomasse aérienne en biomasse souterraine. Ce facteur est égal à 0,24 (Mokany et al., 2006).

\section{Détermination de la biomasse aérienne $\left(B_{a}\right)$}

A cause de la diversité et de la spécificité des formations végétales au sein de la forêt classée de l'Ouémé supérieur, la détermination de la biomasse aérienne a été faite suivant plusieurs modèles allométriques.

\section{$\checkmark$ Pour les espèces de forêt dense et galerie forestière}

L'équation allométrique de Chave et al. (2014) a été utilisée pour l'estimation de la biomasse aérienne des espèces ligneuses présentes dans ces unités de végétation. Cette équation est la suivante :

$$
B_{a f}=0,0673 *\left(\rho D^{2} H\right)^{0,976}
$$

Avec $B_{a f}$ : Biomasse aérienne en $\mathrm{kg}, \mathrm{D}$ : Diamètre mesuré à hauteur de poitrine $(\mathrm{cm}), H$

: Hauteur totale $(m)$ et $\rho$ : Densité spécifique $\left(\mathrm{g} / \mathrm{cm}^{3}\right)$

$\checkmark \quad$ Pour les espèces de forêt claire, de savane et de jachère

L'équation allométrique de Nakou et al. (2016) a été utilisée pour l'estimation de la biomasse forestière des espèces ligneuses présentes dans ces unités de végétation. Elle se présente comme suit :

$B_{a s}=10,1859 * 10^{-5} \times D^{2,1949} \times H^{0,5317} \times \rho^{1,3553}$

$B_{a s}$ : Biomasse aérienne (tonne); $D$ : diamètre

(cm) mesuré à 1,3 m au-dessus du sol, $H$ :

Hauteur totale (m), $\rho$ : Densité spécifique moyenne de l'espèce $\left(t / \mathrm{m}^{3}\right)$

\section{$\checkmark \quad$ Pour certaines espèces en plantation au Bénin}

La biomasse aérienne des espèces comme Tectona grandis et Anacardium occidentale a été calculée en multipliant la biomasse fût par le facteur d'expansion de la biomasse (BEF).

\section{$\checkmark \quad$ Pour l'espèce Tectona grandis}

L'équation proposée par Guendehou et al. (2012) a été utilisée pour estimer la biomasse aérienne des individus de l'espèce. L'équation choisie est de la forme :

$$
\begin{gathered}
B=\exp [-2,1209+0,0409+2,4871 \times \operatorname{Ln}(D)] \\
; B_{a t}=B \times B E F_{t} \text { Avec } \mathrm{BEF}_{\mathrm{t}}=1,38
\end{gathered}
$$

$B_{a t}:$ Biomasse aérienne $(\mathrm{kg}) ; B$ : Biomasse du fût $(\mathrm{kg})$; $D$ : diamètre $(\mathrm{cm})$ mesuré à $1,3 \mathrm{~m}$ audessus du sol

\section{$\checkmark$ Pour l'espèce Anacardium occidentale}

L'équation de Biah et al. (2018) a été utilisée pour l'estimation de la biomasse aérienne. L'équation choisie est de la forme :

$$
\begin{aligned}
& B_{1}=\exp [-1,85645+0,01656+2,02288 \times \operatorname{Ln}(D)] \\
& \quad ; B_{a n}=B_{1} \times B_{E} F_{a} \text { Avec } \mathrm{BEF}_{\mathrm{a}}=1,61
\end{aligned}
$$


$B_{a n}$ : Biomasse aérienne $(\mathrm{kg}) ; B_{1}:$ Biomasse du fût $(\mathrm{kg}) ; D$ : diamètre $(\mathrm{cm})$ mesuré à $1,3 \mathrm{~m}$ au-dessus du sol

\section{Estimation du carbone total}

L'estimation de la quantité du carbone total a été faite à partir du facteur de conversion proposé par Guendehou et al. (2012). Ce facteur de conversion à pour valeur 0,487 (Goussanou et al., 2018). Ainsi, la quantité de carbone total a été déduite de la manière suivante :

$C_{T}=B_{T} \times 0,487$

Avec $\mathrm{C}_{\mathrm{T}}$ : carbone total et $\mathrm{B}_{\mathrm{tT}}$ : biomasse totale Spatialisation de la biomasse forestière et du carbone séquestré

Pour cartographier la biomasse et le carbone, le NDVI a été utilisé comme variable prédictive. Ainsi, les valeurs de NDVI ont été extraites des images landsat OLI-TIRS de 2018. Le modèle puissance a été retenu par ce qu'il présente un pouvoir explicatif plus élevé et la plus faible erreur standard après une implémentation de plusieurs modèles. Les données de NDVI ont subi deux types de transformation. La première transformation (type logarithmique) a permis de faire passer les valeurs de NDVI de l'intervalle $[-1 ; 1]$ à $[0$ ;255]. Ainsi, les valeurs négatives ont pu être évitées dans la manipulation et la visualisation des données (Chabi et al., 2016). La deuxième transformation était celle de Box-Tidwell (1962) pour la détermination du paramètre de forme $\lambda$ suivant la formule :

$$
B=\alpha+\beta^{*} N D V I^{\lambda}+\varepsilon \text { (Modèle }
$$

puissance et ses dérivés)

B : biomasse totale; $\alpha, \beta$ et $\delta$ : coefficients des modèles ; $\varepsilon$ : erreur ; NDVI : Indice de différence normalisé de végétation.

Toutes les analyses ont été exécutées dans le logiciel R 3.5.1 (R Core Team, 2018) et la mise en page cartographique a été faite dans ArcGis 10.3.

Tableau 1 : Nombre de placeaux par unité de végétation.

\begin{tabular}{lc}
\hline Unité de végétation & Nombre de placeaux installés \\
\hline Forêt galerie & 1 \\
Forêt dense sèche & 1 \\
Forêt claire et savane boisée & 23 \\
Savane arborée & 32 \\
Savane arbustive & 9 \\
Plantation & 4 \\
Jachère & 3 \\
\hline Total & $\mathbf{7 3}$ \\
\hline
\end{tabular}




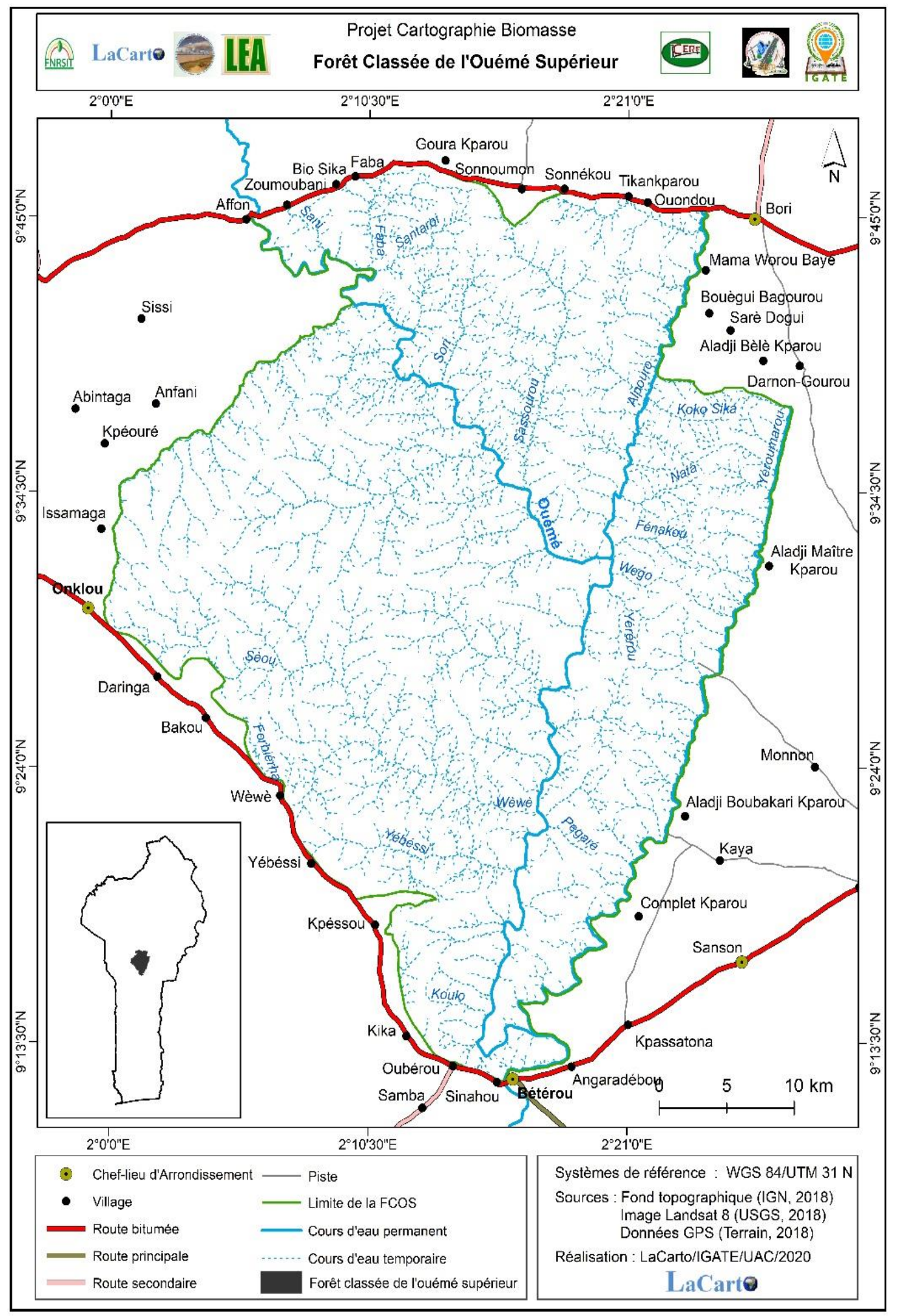

Figure 1 : Localisation géographique de la forêt classée de l’Ouémé supérieur. 


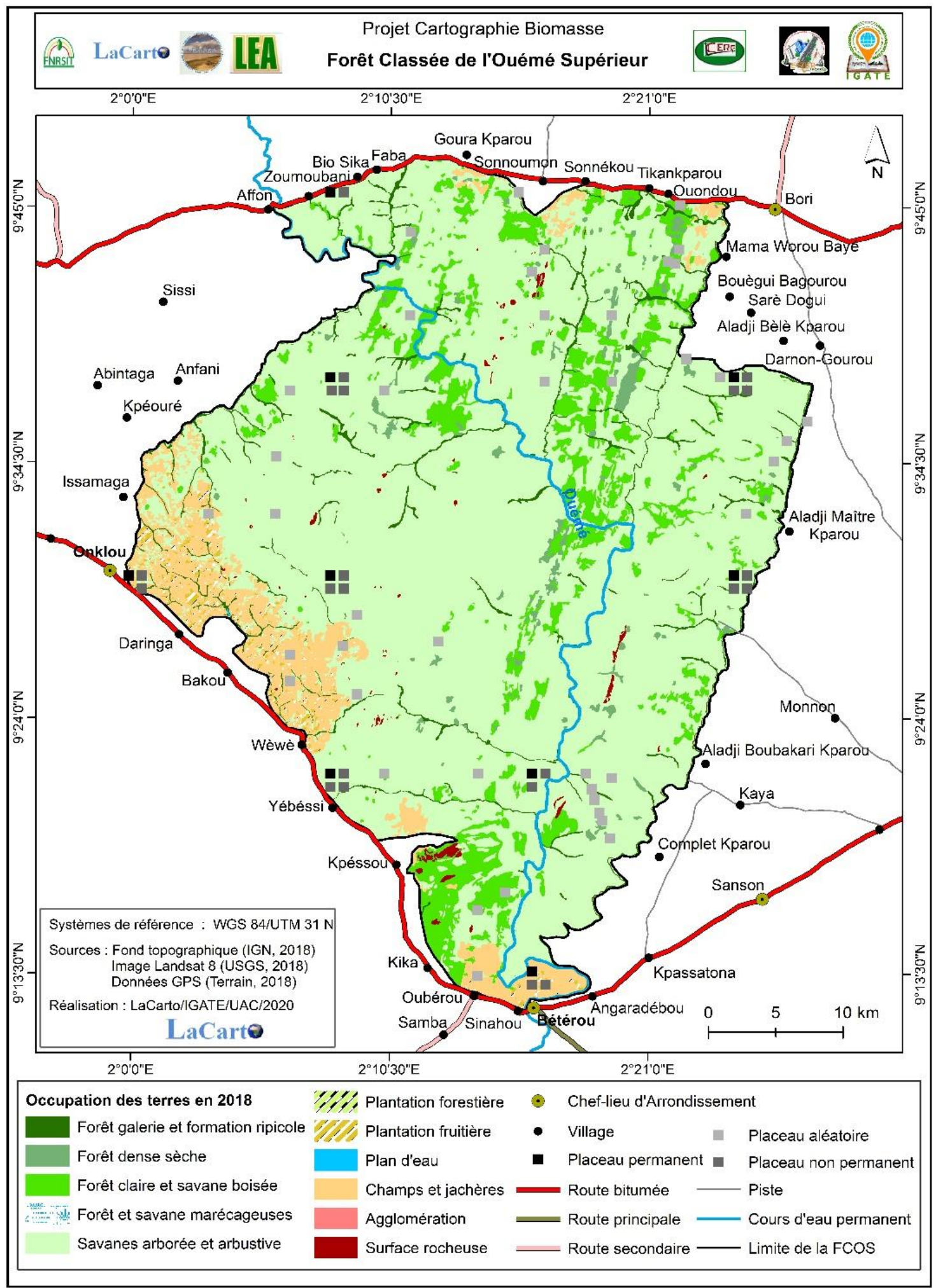

Figure 2 : Spatialisation des placeaux dans de la forêt classée de l'Ouémé supérieur. 


\section{RÉSULTATS}

\section{Biomasse forestière et carbone séquestré par unité de végétation}

Les données d'inventaire ont révélé que la biomasse totale produite par les arbres de la forêt classée de l'Ouémé supérieur avoisine 13 035694 tonnes. La quantité totale de carbone séquestrée a été évaluée à 6348383 tonnes. Le tableau 2 présente les valeurs de biomasse et de carbone par unité de végétation de la forêt classée de l'Ouémé supérieur.

De façon globale, la biomasse forestière totale produite par unité de végétation dans la forêt classée de l'Ouémé supérieur est de 67,54 $\pm 35,25 \mathrm{t} / \mathrm{ha}$ avec une quantité moyenne de carbone séquestré de 32,89 $\pm 17,16$ t/ha. Les formations forestières (forêts galerie, dense sèche et claire) sont celles qui assurent une plus grande production de biomasse et de séquestration de carbone dans la forêt classée de l'Ouémé supérieur (Tableau 2). Parmi les formations savanicoles, seules les savanes boisées accumulent une quantité de biomasse $(89,53 \pm 24,55$ t/ha) largement au-dessus de la moyenne $(67,54 \pm 35,25 \mathrm{t} / \mathrm{ha})$ notée pour l'ensemble de la forêt classée (Tableau 2).
Cartographie de la biomasse et du carbone de la forêt classée de l'Ouémé supérieur

La spatialisation de la biomasse et du carbone de la forêt a été faite en utilisant comme fonction de lien un modèle de régression de la forme :

$$
\begin{aligned}
& B=-135,50+0,0326 * N D V I^{1,75} \\
& \left(R^{\mathbf{2}}=68,48 \% \quad p=2,2 * 10^{-16}\right)
\end{aligned}
$$

Le pouvoir explicatif du modèle de prédiction de la biomasse forestière et du carbone en fonction du NDVI indique qu'environ $68 \%$ de la variation de la biomasse et du carbone dans la forêt classée de l'Ouémé supérieur sont expliquées par la variation des valeurs de NDVI. La distribution spatiale de la biomasse et du carbone a montré une prédominance de plage de fortes quantités de biomasse et de carbone dans le centre-ouest et surtout dans les forêts galeries (Figures 3 et 4). Les périphéries ouest, sud, sud-ouest et nord sont caractérisées par de faibles valeurs de biomasse (Figure 3) et séquestrent ainsi moins de carbone (Figure 4) par rapport aux autres secteurs de ladite forêt.

Tableau 2 : Valeurs de biomasse et de carbone par unité de végétation de la forêt classée de l'Ouémé supérieur.

\begin{tabular}{lccccc}
\hline $\mathbf{U V}$ & & $\mathbf{B a}(\mathbf{t} / \mathbf{h a})$ & $\mathbf{B r}(\mathbf{t} / \mathbf{h a})$ & $\mathbf{B}_{\mathbf{T}}(\mathbf{t} / \mathbf{h a})$ & $\mathbf{C}_{\mathbf{T}}(\mathbf{t} / \mathbf{h a})$ \\
\hline \multirow{2}{*}{ Forêt galerie } & $m$ & 172,200 & 41,328 & 213,529 & 103,988 \\
& $\sigma$ & - & - & - & - \\
\hline \multirow{2}{*}{ Forêt dense sèche } & $m$ & 79,841 & 19,162 & 99,002 & 48,214 \\
& $\sigma$ & - & - & - & - \\
\hline \multirow{2}{*}{ Forêt claire } & $m$ & 93,117 & 22,348 & 115,465 & 56,231 \\
& $\sigma$ & 29,098 & 6,984 & 36,082 & 17,572 \\
\hline \multirow{2}{*}{ Savane boisée } & $m$ & 72,198 & 17,327 & 89,525 & 43,599 \\
& $\sigma$ & 19,800 & 4,752 & 24,552 & 11,957 \\
\multirow{2}{*}{ Savane arborée } & $m$ & 46,056 & 11,053 & 57,109 & 27,812 \\
& $\sigma$ & 12,434 & 2,984 & 15,418 & 7,508 \\
\multirow{2}{*}{ Savane arbustive } & $m$ & 27,615 & 6,628 & 34,243 & 16,676 \\
& $\sigma$ & 9,144 & 2,195 & 11,339 & 5,522 \\
\hline \multirow{2}{*}{ Jachère } & $m$ & 32,437 & 7,785 & 40,222 & 19,588 \\
& $\sigma$ & 12,522 & 3,005 & 15,527 & 7,562 \\
\multirow{2}{*}{ Plantation } & $m$ & 34,882 & 8,372 & 43,253 & 21,064 \\
& $\sigma$ & 31,443 & 7,546 & 38,990 & 18,988 \\
\multirow{2}{*}{ Global } & $m$ & 54,470 & 13,073 & 67,543 & 32,894 \\
& $\sigma$ & 28,424 & 6,822 & 35,245 & 17,164
\end{tabular}

$\mathrm{Ba}:$ Biomasse aérienne $; \mathrm{Br}:$ Biomasse racinaire $; \mathrm{B}_{\mathrm{T}}:$ Biomasse totale $; \mathrm{C}_{\mathrm{T}}:$ Carbone total $; m:$ moyenne $; \sigma:$ Ecarttype. 


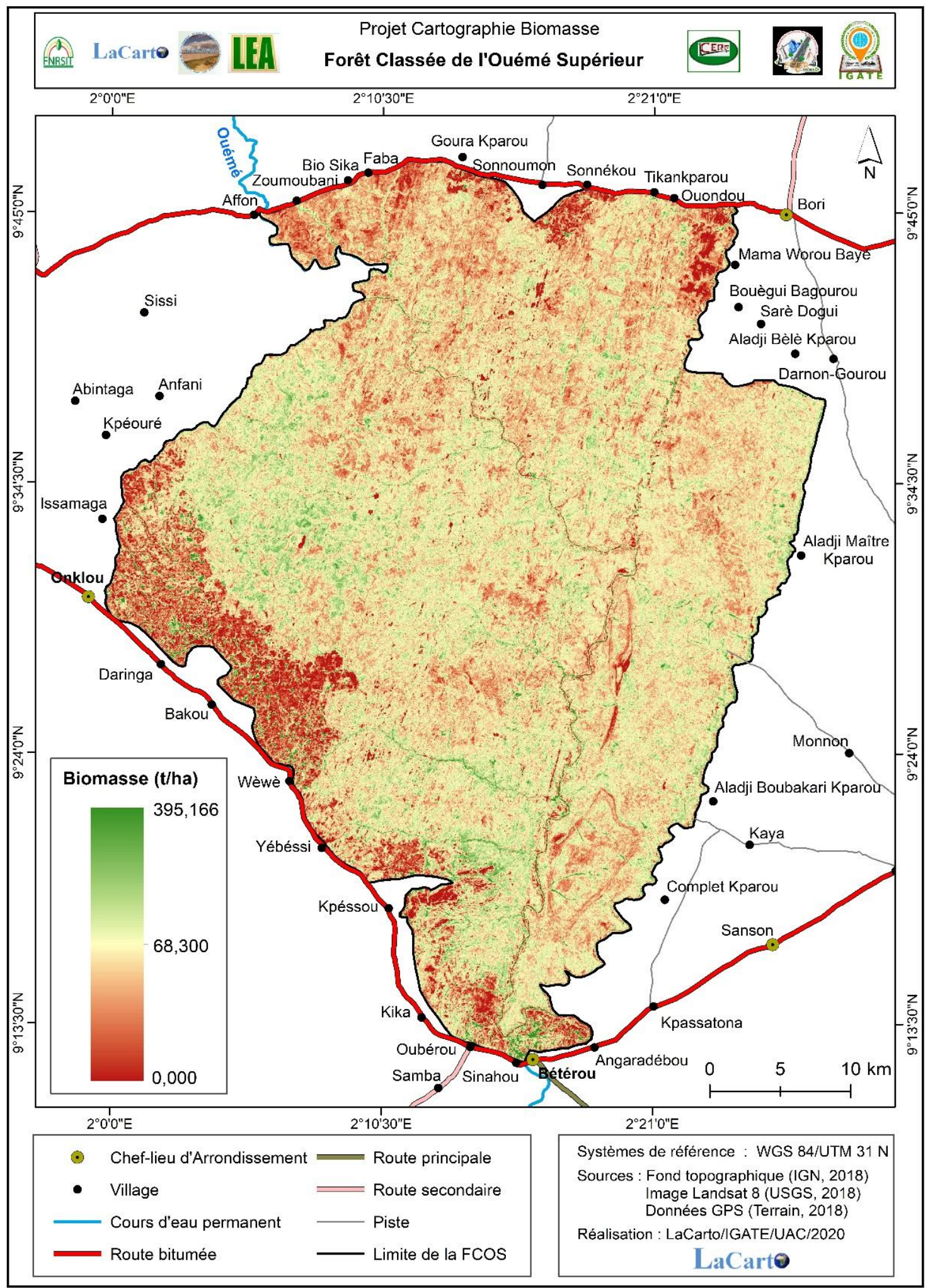

Figure 3 : Distribution de la biomasse dans la forêt classée de l'Ouémé supérieur. 


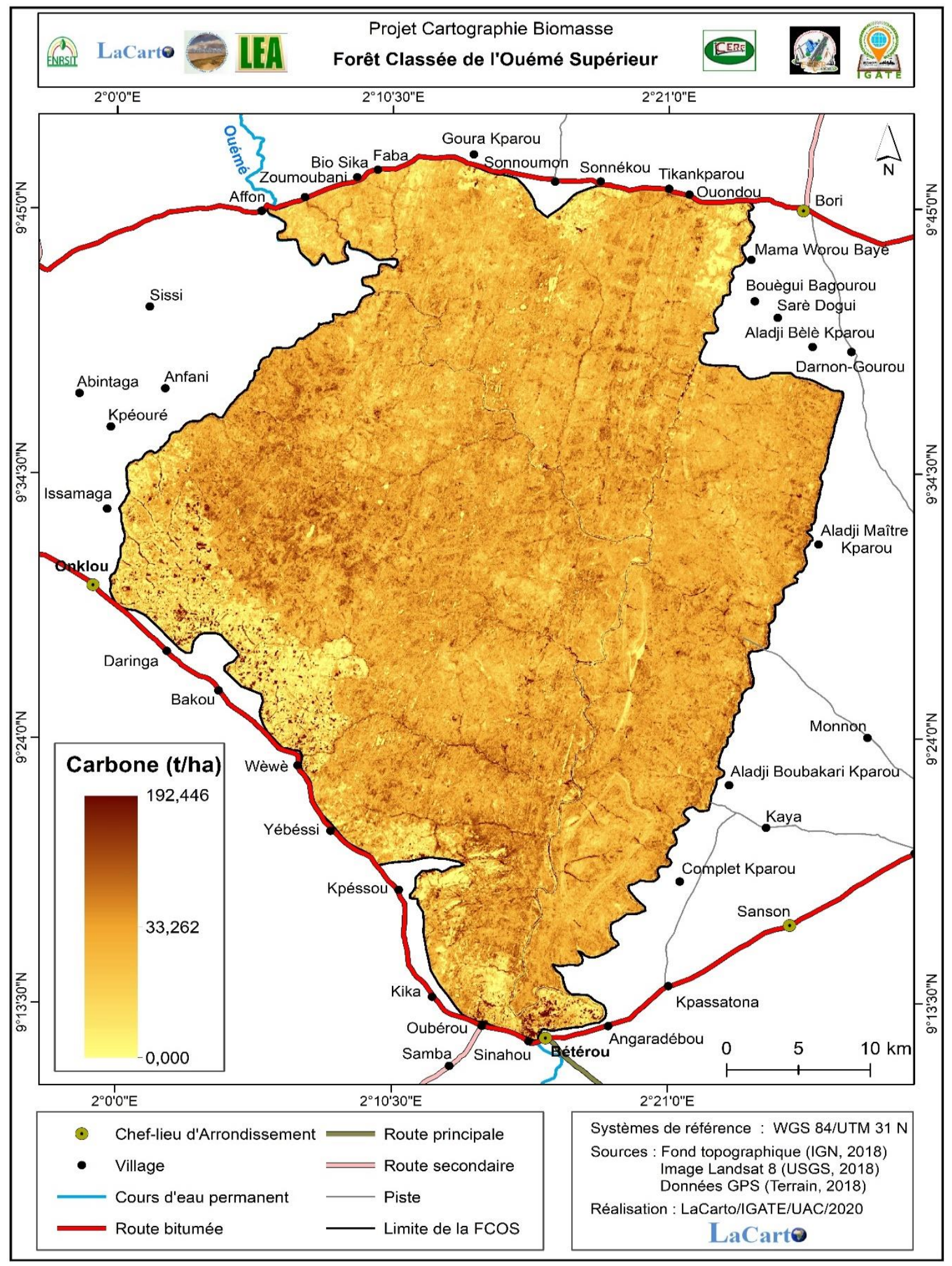

Figure 4 : Spatialisation du carbone séquestré dans la forêt classée de l'Ouémé supérieur. 


\section{DISCUSSION}

\section{Biomasse forestière et du carbone séquestré de la forêt classée de l'Ouémé supérieur}

La présente recherche a utilisé la méthode non destructive pour l'estimation de la biomasse forestière et le stock de carbone à travers des équations allométriques. Cette méthode est la plus utilisée en matière de quantification de la biomasse forestière et du stock de carbone (Bakayoko et al., 2012; Issifou Moumouni et al., 2017 ; Adamou et al., 2020 ; Adjéran et al., 2020). Elle présente l'avantage d'être rapidement opérationnelle en termes de temps et de coût d'estimation. Cependant, plusieurs auteurs ont souligné que l'utilisation de ces équations allométriques en dehors des sites pour lesquels elles ont été développées pourrait entrainer des biais au niveau des estimations (Chave et al., 2004 ; Molto et al., 2013 ; Loubota Panzou et al., 2016). La quantité totale de carbone séquestré par les arbres de la forêt classée de l'Ouémé supérieur est évaluée à 6348 382,834 tonnes. Cette quantité est largement inférieure au stock de carbone obtenu (24 086 528,6 tonnes) dans le massif forestier de Ngog-Mapubi au Cameroun par Ngoufo et al. (2019). La différence observée peut s'expliquer par la variation de la méthode d'estimation utilisée. La quantité de biomasse forestière et de carbone séquestré obtenus varient suivant les types de formation végétale du milieu d'étude. Cette observation est similaire à celle de Ouédraogo et al. (2019) dans le massif forestier de Dindéresso au Burkina-Faso. Cette variation serait liée aux paramètres structuraux intrinsèques propres à chaque unité de végétation. Selon plusieurs auteurs, la biomasse forestière et le stock de carbone dépendent de plusieurs facteurs qui sont entre autres la qualité, la quantité et la contribution des individus de gros diamètre (Joosten et al., 2004 ; Amougou et al., 2016). De façon globale on note une variation décroissante de la biomasse forestière et du carbone séquestré des formations forestières vers celles savanicoles et post-culturales.

\section{Spatialisation de la biomasse forestière et du carbone séquestré}

Le pouvoir explicatif du modèle puissance utilisé indique que $68 \%$ de la variation de la biomasse forestière et du carbone séquestré dans la forêt classée de l'Ouémé supérieur sont dues à la variation des valeurs de NDVI. Par contre, $32 \%$ de cette variation sont liés à d'autres facteurs non pris en compte par le modèle. Ce pouvoir prédictif est inférieur à celui obtenu par Avitabile et al. (2012) avec un taux de $81 \%$ en Ouganda. La différence de résultats serait liée au type de modèle utilisé pour les estimations. Ces résultats mettent en relief, le rôle déterminant du NDVI dans la cartographie de la biomasse forestière et $\mathrm{du}$ carbone séquestré. $\mathrm{La}$ distribution spatiale de la biomasse et du carbone montre de fortes quantités de biomasse et de carbone séquestré à l'intérieur de la forêt classée qu'aux niveaux des zones périphériques surtout les zones qui sont situées le long des routes. Les faibles valeurs obtenues au niveau de la zone périphérique indiquent que ces zones sont caractérisées par de faibles valeurs de NDVI et sont par conséquent plus dégradées que l'intérieur de la forêt.

\section{Conclusion}

La présente recherche a permis de noter que la biomasse forestière et le stock de carbone varient suivant les unités de végétation de la forêt classée de l'Ouémé supérieur au Centre-Bénin. La quantité de biomasse et de carbone séquestrée est plus importante dans les forêts galeries, les forêts claires, les forêts denses sèches et les savanes boisées contrairement aux savanes arboré et arbustive, aux plantations et aux jachères. Ces résultats permettent d'affirmer que la biomasse forestière et le stock de carbone évoluent suivant un gradient décroissant des formations naturelles plus stables vers celles perturbées. La distribution spatiale de la biomasse et du carbone montrent de fortes quantités de biomasse et de carbone séquestré à l'intérieur de la forêt classée qu'aux niveaux des zones périphériques surtout les zones qui sont situées le long des routes. Ces résultats suggèrent la mise en place par l'administration forestière, d'une politique de la restauration des formations plus dégradées afin d'accroitre le potentiel de la biomasse forestière et du carbone séquestré dans ladite forêt pour une contribution considérable à l'atténuation des effets du changement climatique dans le 
processus de réduction des émissions issues de la déforestation et de la dégradation des écosystèmes forestiers au Bénin.

\section{CONFLIT D'INTÉRÊTS}

Les auteurs déclarent qu'ils n'ont aucun conflit d'intérêt lié à cet article.

\section{CONTRIBUTIONS DES AUTEURS}

Tous les auteurs ont participé à la conception et à la planification de l'étude, puis à la collecte et au traitement des données. SK a rédigé le premier draft du manuscrit. $\mathrm{MD}, \mathrm{OA}$, SZ, AOM, ITI, IY, JD ont participé à la correction du premier draft du manuscrit. $\mathrm{CH}$ et OM ont supervisé les travaux de collecte, de traitement des données et de rédaction du manuscrit.

\section{RÉFÉRENCES}

Adamou S, Amani A, Mahamadou HM, Yaye AD. 2020. Modèle allométrique d'estimation du carbone aérien séquestré par Balanites aegyptiaca (L.) Del dans la partie Sud-Ouest du Niger. Afrique Science, 16(6): 188-203.

Adjéran RW, Lougbegnon TO, Djego GJ. 2020. Estimation de la biomasse aérienne et du carbone des arbres de Lophira lanceolata dans la forêt classée de l'Ouémé supérieur au Bénin. Afrique Science, 16(5): 17-31.

Adomou AC. 2011. Phytogéographie du Bénin: 14-18. In Protection de la Nature en Afrique de l'Ouest: Une Liste Rouge pour le Bénin, Neuenschwander P, Sinsin $B$, Georgen G (eds). International Institute of Tropical Agriculture: Ibadan, Nigeria; 36-45.

Ajonina GN, Ago EE, Amoussou G, Mibog ED, Akambi ID, Dossa E. 2014. Carbon budget as a tool for assessing mangrove forests degradation in the western, coastal wetlands complex (Ramsar Site 1017) of Southern Benin, West Africa. In The Land/Ocean Interactions in the Coastal Zone of West and Central Africa. Springer : Cham ; 139-149.

Amougou JA, Bembong Ebokona LD, Batha RAS, Mala AW, Ngono H. 2016. Estimation du stock de carbone dans deux unités de terre en zone de savane du
Cameroun. Revue de Géographie, d'Aménagement Régional et $d u$ Développement des Suds, Regardsuds, 2 : 28-45.

Avitabile V, Baccini A, Friedl MA, Schmullius C. 2012. Capabilities and limitations of Landsat and land cover data for aboveground woody biomass estimation of Uganda. Remote Sensing of Environment, 117: 366-380.

Bakayoko O, Assa AM, Coulibaly B, N'Guessan KA. 2012. Stockage de Carbone dans des peuplements de Cedrela Odorata et de Gmelina Arborea en Côte d'Ivoire. European Journal of Scientific Research, 75(4): 490-501.

Bastin JF, Barbier N, Couteron P, Adams B, Shapiro A, Bogaert J, De Cannière C. 2014. Aboveground biomass mapping of African forest mosaics using canopy texture analysis: toward a regional approach. Ecological

Applications, 24(8): 1984-2001.

Biah I, Guendehou S, Goussanou C, Kaire M, Sinsin AB. 2018. Allometric models for estimating biomass stocks in cashew (Anacardium occidentale L.) plantation in Benin. Bulletin de la Recherche Agronomique du Bénin, $84: 16$ - 27.

Box GE, Tidwell PW. 1962. Transformation of the independent variables. Technometrics, 4(4): 531-550.

Brown S, Gillespie AJ, Lugo AE. 1989. Biomass estimation methods for tropical forests with applications to forest inventory data. Forest Science, 35(4): 881-902.

Chabi A, Lautenbach S, Orekan VOA, KyeiBaffou N. 2016. Allometric models and aboveground biomass stocks of a West African Sudan Savannah watershed in Benin. Carbon Balance Manage, 11(1):118. DOI: 10.1186/s13021-016-0058-5

Chave J, Mechain MR, Burquez A, Chidumayo E, Colgan MS, Delitti WBC, Duque A, Eid T, Fearnside PM, Goodman RC, Henry M, Martinez-Yrizar A, Mugasha WA, Muller-Landau HC, Mencuccini M, Nelson BW, Ngomanda A, Nogueira EM, Ortiz-Malavassi E, Pelissier R, Ploton P, Ryan CM, Saldarriaga JG, Vieilledent G. 2014. Improved allometric models to 
estimate the aboveground biomass of tropical trees. Global Change Biology, 20(10): 3177-3190. DOI: 10.1111/gcb.12629

Chave J, Condit R, Aguilar S, Hernandez A, Lao S, Perez R. 2004. Error propagation and scaling for tropical forest biomass estimates. Philosophical Transactions of the Royal Society of London: Biological Sciences, 359(1443): 409-420.

Clark D, Brown S, Kicklighter D, Chambers J, Thomlinson J, Ni J, Holland E. 2001. Net primary production in tropical forests: an evaluation and synthesis of existing field data. Ecological Applications, 11(2): 371384. DOI: https://doi.org/10.1890/10510761

Clark DB, Kellner JR. 2012. Tropical forest biomass estimation and the fallacy of misplaced concreteness. Journal of Vegetation Science, 23(6): 1191-1196. DOI : $10.1111 / \mathrm{j} .1654$ 1103.2012.01471.x

FAO. 2009. La situation mondiale de l'alimentation et de l'agriculture. FAO, Rome, 202 p.

Fayolle A, Doucet JL, Gillet JF, Bourland N, Lejeune P. 2013. Tree allometry in Central Africa: Testing the validity of pantropical multi-species allometric equations for estimating biomass and carbon stocks. Forest Ecology and Management, 305: 29-37. DOI: 10.1016/j.foreco.2013.05.036

Feldpausch TR, Lloyd J, Lewis SL, Brienen RJ, Gloor M, Monteagudo Mendoza A, Phillips OL. 2012. Tree height integrated into pantropical forest biomass estimates. Biogeosciences, 9(8): 33813403.

GIEC. 2003. Recommandations du GIEC en matière de bonnes pratiques pour le secteur de l'utilisation des terres, changements d'affectation des terres et foresterie. GIEC, Kanagawa, Japon, 25 p.

Goetz SJ, Baccini A, Laporte NT, Johns T, Walker W, Kellndorfer J, Sun M. 2009. Mapping and monitoring carbon stocks with satellite observations: a comparison of methods. Carbon Balance and Management, 4(1): 1-7. DOI: https://doi.org/10.1186/1750-0680-4-2
Goussanou CA, Guendehou S, Assogbadjo AE, Sinsin B. 2018. Application of sitespecific biomass models to quantify spatial distribution of stocks and historical emissions from deforestation in a tropical forest ecosystem. Journal of Forestry Research, 29(1): 205-213. DOI: 10.1007/s11676-017-0411

Guendehou GHS, Lehtonen A. 2014. Guidance for tree measurement in tropical forest ecosystems using non-destructive sampling to develop stem biomass and volume models. Working Papers of the Finnish Forest Research Institute, Metlan työraportteja.

Guendehou GHS, Lehtonen, Moudachirou M, Mäkipää R, Sinsin B. 2012. Stem biomass and volume models of selected tropical tree species in West Africa. Southern Forests, $\quad \mathbf{7 4}(2)$ : $\quad 77-88 . \quad$ DOI: 10.2989/20702620.2012.701432

Houghton RA. 2005. Aboveground forest biomass and the global carbon balance. Global Change Biology, 11(6): 945-958.

IPCC. 2007. Guidelines for National Greenhouse Gas Inventories. In Agriculture, Forestry and Other Land Use. IGES Publisher, Japan.

Moumouni YI, Arouna O, Issaka NT, Imorou IT, Zakari S, \& Djaouga M. 2017. Estimation de la variabilité de la biomasse aérienne ligneuse en forêt tropicale sèche: cas de la forêt classée de Wari-Maro au Centre-Bénin. Revue de Géographie du Laboratoire Leïdi, 17: 38-56.

Joosten R, Schumacher J, Wirth C, Schulte A. 2004. Evaluating tree carbon predictions for beech (Fagus sylvatica L.) in western Germany. Forest Ecology And Management, 189(1-3): 87-96.

Kooke GX, Ali RKFM, Djossou JM, Imorou IT. 2019. Estimation du stock de carbone organique dans les plantations de Acacia auriculiformis A. Cunn. ex Benth. des forêts classées de Pahou et de Ouèdo au Sud du Bénin. International Journal of Biological and Chemical Sciences, 13(1): 277-293. DOI: 10.4314/ijbcs.v13i1.23

Kuyah S, Dietz J, Muthuri C, Jamnadass R., Mwangi P, Coe R, Neufeldt H. 2012. Allometric equations for estimating 
biomass in agricultural landscapes: Belowground biomass. Agriculture, Ecosystems \& Environment, 158: 225234. DOI: $10.1016 /$ j.agee.2012.05.010

IFN. 2006. Méthodologie et instructions pour l'exécution des inventaires forestiers national et régional du Bénin, Projet Bois de Feu, phase II. Ministère de l'Environnement et de la Protection de la Nature, Cotonou, Bénin, 55 p.

Fagerholt K, Laporte G, Norstad I. 2010. Reducing fuel emissions by optimizing speed on shipping routes. Journal of the Operational Research Society, 61(3): 523-529.

DOI: https://doi.org/10.1057/jors.2009.77

Laporte N, Baccini A, Goetz S, Mekui P, Bausch A. 2010. Une première estimation de la biomasse ligneuse aérienne d'Afrique sur la base d'images satellites et d'inventaire forestiers. Conference on Carbon stock and fluxes, COPIFAC, Brazzaville, 58-65.

Loubota Panzou GJ, Doucet JL, Loumeto JJ, Biwole A, Bauwens S, Fayolle A. 2016. Biomasse et stocks de carbone des forêts tropicales africaines (synthèse bibliographique). Biotechnologie,

Agronomie, Société et

Environnement, 20(4): 508-522.

Mokany K, Raison RJ, Prokushkin AS. 2006. Critical analysis of root: shoot ratios in terrestrial biomes. Global Change Biology, 12(1): 84-96. DOI: https://doi.org/10.1111/j.13652486.2005.001043.x

Molto Q, Rossi V, Blanc L. 2013. Error propagation in biomass estimation in tropical forests. Methods in Ecology and Evolution, 4(2): 175-183.

Mugasha WA, Eid T, Bollandsås OM, Malimbwi RE, Chamshama SAO, Zahabu E, Katani JZ. 2013. Allometric models for prediction of above-and belowground biomass of trees in the miombo woodlands of Tanzania. Forest Ecology and Management, 310: 87-101.

Nakou A, Sauter UH, Kohnle U. 2016. Improved models of harvest-induced bark damage. Annals of Forest Science, 73(2): 233-246.

Ngomanda A, Obiang NLE, Lebamba J, Mavouroulou QM, Gomat H, Mankou GS, Picard N. 2014. Site-specific versus pantropical allometric equations: Which option to estimate the biomass of a moist central African forest? Forest Ecology and Management, 312: 1-9. DOI: https://doi.org/10.1016/j.foreco.2013.10. 029

Ngoufo R, Zapfack L, Dongfack ET, Ngoufo LST, Guimdo CAM, Aurélien C, Njal S. 2019. Évaluation et spatialisation du carbone stocké dans le massif forestier de Ngog-Mapubi (Cameroun). Conférence OSFACO: Des images satellites pour la gestion durable des territoires en Afrique, Brazzaville. hal-02189496.

Ouedraogo WO, Gomgnimbou AP, Santi S, Ilboudo D, Toguyeni A. 2019. Quantification de la Biomasse et stockage du carbone du massif forestier de l'Ecole Nationale des Eaux et Forêts de Dindéresso province du Houet au Burkina Faso. International Journal of Biological and Chemical Sciences, 13(7): 32763288. DOI: $10.4314 /$ ijbcs.v13i7.24

Pascal C, Peter S. 2015. Climat: 30 Questions Pour Comprendre la Conférence de Paris. Éditions Les Petits Matins : Paris.

$\mathrm{R}$ Core Team. 2018. R: A language and environment for statistical computing. $\mathrm{R}$ Foundation for Statistical Computing, Vienna, Austria. http://www.Rproject.org/

Saïdou A, Dossa AFE, Gnanglè PC, Balogoun I, Aho N. 2012. Evaluation du stock de carbone dans les systèmes agroforestiers à karité (Vitellaria paradoxa CF Gaertn.) et à néré (Parkia biglobosa Jacq. G. Don) en zone Soudanienne du Bénin. Bulletin de la Recherche Agronomique du Bénin, 1-9.

White F. 1986. La végétation d'Afrique. Mémoire accompagnant la carte de la végétation d'Afrique. Office de la Recherche Scientifique et Technique d'Outre-Mer, United Nations, 391 p. 